\section{CDC'S 2009 H1N1 VACCINE \\ PHARMACY INITIATIVE \\ IN THE UNITED STATES: \\ IMPLICATIONS FOR FUTURE \\ PUBLIC HEALTH AND PHARMACY \\ COLLABORATIONS \\ FOR EMERGENCY RESPONSE}

\section{To the Editor}

During the 2009 H1N1 influenza pandemic, the CDC contacted the 50 state, New York City, and District of Columbia health departments and the health department in Puerto Rico through the Association of State and Territorial Health Officials (ASTHO), to discuss distributing 2009 H1N1 influenza vaccine directly to large pharmacy chains ("pharmacies") to supplement state vaccination efforts. By the end of December 2009 , most states had opened vaccination to all members of the public and a vaccine surplus was projected. All but three states opted to take part in this CDC 2009 H1N1 Vaccine Pharmacy Initiative.* The CDC subsequently invited the largest 15 US pharmacies (by prescription share) to participate, 12 of these pharmacies expressed interest and 10 ultimately participated. ${ }^{1}$ From December 2009-February 2010, the CDC distributed 5483900 doses of 2009 $\mathrm{H} 1 \mathrm{~N} 1$ vaccine to these pharmacy chains; they in turn, distributed it to more than 10700 retail stores nationwide. The amount of $2009 \mathrm{H} 1 \mathrm{~N} 1$ vaccine that the CDC directly distributed to pharmacy chains comprised approximately $23 \%$ of all vaccine distributed during the same time period to the same states and accounted for approximately $4.3 \%$ of all 2009 H1N1 vaccine distributed during October 2009. February 2010. Approximately $10 \%$ of adults who received 2009 H1N1 influenza vaccine reported getting vaccinated at a pharmacy. ${ }^{2}$ This included vaccinations given with vaccine provided to pharmacies by state health officials and directly by the CDC (Figure).

\footnotetext{
*Georgia, Hawaii, and West Virginia did not participate for a variety of reasons including ongoing distribution of H1N1 vaccine to pharmacies in the state through the health department. Puerto Rico (the only US insular area with retail locations of the pharmacy chains) also opted not to participate.
}

The CDC conducted assessments with key contacts at each participating pharmacy chain (by telephone with each of the pharmacies in February 2010 and in-person in March 2010) and held a debriefing with local and state public health officials in February 2010. Pharmacies reported that the majority of their locations $(>50 \%)$ had received little or no vaccine from states prior to the beginning of the Initiative, likely due to scarcity of vaccine and diverse approaches to distribution. Differing accountability and data reporting requirements across jurisdictions (including at times, multiple reporting requirements within a given state), created an administrative burden for national pharmacies aggregating data at the corporate level. Some pharmacy chains also reported that insurers (such as Medicaid) in some states denied requests for reimbursement for administration fees for vaccinations given in pharmacy settings. States expressed concern over lack of awareness of the number of doses of vaccine distributed by pharmacies into their state; it was important for state and local public health officials to be able to accurately track 2009 H1N1 influenza vaccine available to their populations.

Pharmacies were able to play an important role in the $2009 \mathrm{H} 1 \mathrm{~N} 1$ influenza vaccination effort because of their experience vaccinating adults against influenza. ${ }^{3}$ As of July 2009, all 50 states allow pharmacists to administer influenza vaccines under prescribing protocols, but these vary by patient age (pharmacists can vaccinate adults against influenza in all 50 states). ${ }^{4}$ ASTHO developed a framework for formalizing partnerships between pharmacies and public health for administering 2009 H1N1 vaccine that was useful in rapidly establishing the Initiative. $^{5}$

Pharmacies offer expertise, convenience, accessibility, extended hours of operation, and can be integrated as key community partners early in a public health response. By leveraging pharmacies' systems for national, regional, and local storage and distribution, and ability to administer vaccines on a large scale, more 2009 H1N1 influenza vaccine was made accessible at the community level. The Initiative also 


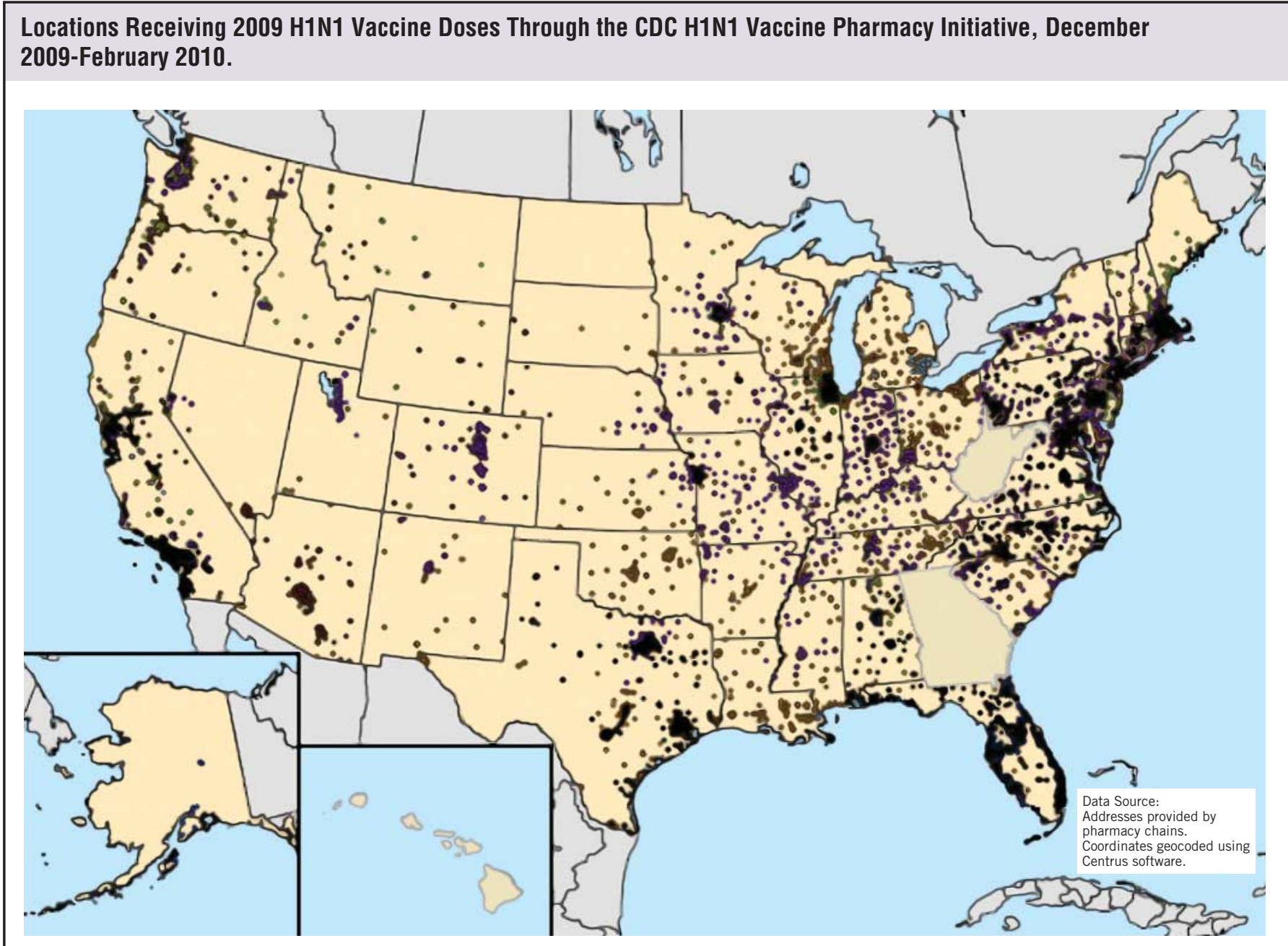

NOTE: Georgia, Hawaii, and West Virginia did not participate for a variety of reasons, including ongoing distribution of H1N1 vaccine with pharmacies in the state through the health department.

expanded public health understanding of pharmacy capabilities to offer vaccines and potentially other countermeasures during public health emergencies. ${ }^{6}$

\section{Lisa M. Koonin, MN, MPH}

Denise R. Beauvais, MS Tom Shimabukuro, MD, MPH, MBA Pascale M. Wortley, MD, MPH Jane B. Palmier, JD

Toscha R. Stanley, MSA John Theofilos, PT, MBA, BS Toby L. Merlin, MD

Author Affiliations: Ms Koonin is Senior Advisor, Influenza Coordination Unit, Office of Infectious Diseases, Centers for Disease Control and Prevention; Ms Beauvais is Public Health Analyst, Influenza Coordination Unit, Office of Infectious Diseases, Centers for Disease Control and Prevention; Dr Shimabukuro is Senior Medical Officer, Immunization Safety Office,
Centers for Disease Control and Prevention; Dr Wortley is Chief, Health Services Research \& Evaluation Branch, Immunization Services Division, National Center for Immunization and Respiratory Diseases, Centers for Disease Control and Prevention; Ms Palmier is with the Institute of Public Health, Georgia State University; Atlanta, Georgia; Ms Stanley is Health Services Officer, National Center for Immunization and Respiratory Diseases, Centers for Disease Control and Prevention; $\mathrm{Mr}$ Theofilos is Lead, Capacity Building, Training, and Technical Assistance, Office of the Director, Division of State and Local Readiness, Office of Public Health Preparedness and Response, Centers for Disease Control and Prevention; Dr Merlin is Director, Division of Preparedness and Emerging Infections, National Center for Emerging and Zoonotic Infections, Centers for Disease Control and Prevention.

Correspondence: Lisa M. Koonin, MN, MPH, Office of Infectious Diseases, Centers for Disease Control and Prevention, 1600 Clifton Road, NE MS A-20, Atlanta, GA 30333 (e-mail: LKoonin@cdc.gov).

Author Disclosures: No nongovernmental funds were received to conduct this work. The authors report no conflicts of interest. 
Acknowledgments: We would like to acknowledge the important contributions of Stephen Redd, Paul Jarris, Jim Blumenstock, Isaac Weisfuse, Jack Hermann, and Jeanne Santoli to the success of this initiative. We would also like to recognize essential collaborations with the National Association of Chain Pharmacies and its members, the participating pharmacy chains, and the American Pharmacists Association for making this emergency effort possible.

\section{REFERENCES}

1. NACDS. 2009-2010 Chain Pharmacy Industry Profile. 2009. Alexandria, VA: National Association of Chain Drug Stores; 2009.

2. C.S. Mott Children's Hospital. the University of Michigan Department of Pediatrics and Communicable Diseases, and the University of Michigan Child Health Evaluation and Research (CHEAR) Unit. Health Care Provider Recommendations Key to H1N1 Vaccination. Vol. 9 Issue 1. January 26, 2010. https://www.med.umich.edu/mott/npch/pdf/012610report .pdf. Accessed April 8, 2011.
3. Hogue MD, Grabenstein JD, Foster SL, Rothholz MC. Pharmacist involvement with immunizations: a decade of professional advancement. J Am Pharm Assoc (2003). 2006;46(2):168-179, quiz 179-182.

4. American Pharmacists Association. States Where Pharmacists Can Immunize. Washington (DC): American Pharmacists Association; 2009. http://www .pharmacist.com/AM/Template.cfm?Section=Pharmacist_Immunization _Center $1 \&$ TEMPLATE=/CM/ContentDisplay.cfm\&CONTENTID $=21623$. Accessed April 8, 2011.

5. Association of State and Territorial Health Officials. Operational Framework for Partnering with Pharmacies for Administration of 2009 H1N1. Arlington (VA): Association of State and Territorial Health Officials; 2009. http://www.astho.org/Display/AssetDisplay.aspx?id=2613. Accessed April 8, 2011.

6. Stroud C, Nadig L, Altevogt BM. Forum on Medical and Public Health Preparedness for Catastrophic Events. Medical Countermeasures Dispensing: Emergency Use Authorization and the Postal Model: Workshop Summary. Washington, DC: Institute of Medicine; 2010. 